\title{
Job, Hubert; Mayer, Marius; Haßlacher, Peter; Nischik, Gero; Knauf, Christoph; Pütz, Marco; Essl, Josef; Marlin, Andreas; Kopf, Manfred; Obkircher, Stefan (2017): Analyse, Bewertung und Sicherung alpiner Freiräume durch Raumordnung und räumliche Planung
}

\author{
Hannover: Akademie für Raumforschung und Landesplanung. = Forschungsberichte \\ der ARL 7. 88 Seiten, 10 Tabellen, 9 Abbildungen
}

\section{Werner Bätzing 1}

Online publiziert: 25. Juli 2017

(c) Springer-Verlag GmbH Deutschland 2017

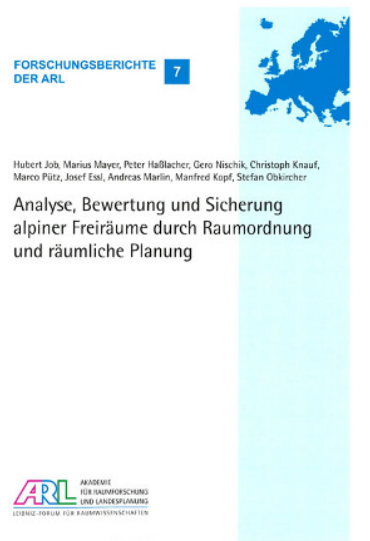

\section{Ausgangssituation und Leitfrage}

Die Alpen werden seit längerer Zeit in allen Höhenlagen immer intensiver von verschiedensten Interessengruppen genutzt (Land- und Forstwirtschaft, Gewerbe, Industrie, Wasserkraft, Energiewirtschaft, Tourismus, Verkehr, Wohnen). Dabei werden immer mehr ehemalige Freiräume mittels technischer Infrastrukturen erschlossen und zerschnitten, was nicht nur die Bedeutung der Alpen als vielfältiges Ökosystem und als attraktive Landschaft beeinträchtigt, sondern was sich auch negativ auf eine nachhaltige Landund Forstwirtschaft, auf einen naturnahen Tourismus so-

Prof. em. Dr. Werner Bätzing

werner.baetzing@web.de

1 Archiv für integrative Alpenforschung,

Geyerswörthstraße 12, 96047 Bamberg, Deutschland wie auf die regionale Identität auswirkt (S. 1 f.). Daraus erwachsen die Aufgabe und die Leitfrage des vorliegenden Bandes, wie die noch bestehenden Freiräume in Zukunft angemessen gesichert werden können.

\section{Normative Leitideen für die Bearbeitung der Leitfrage}

Um diese Leitfrage zu beantworten, bildete sich innerhalb der Akademie für Raumforschung und Landesplanung (ARL) eine Arbeitsgruppe. Diese bestand aus Wissenschaftlern (die Geographieprofessoren Hubert Job (Würzburg) und Marius Mayer (Greifswald) mit den beiden Mitarbeitern Gero Nischik und Christoph Knauf, erweitert durch Marco Pütz von der Eidgenössischen Forschungsanstalt für Wald, Schnee und Landschaft (Birmensdorf/Schweiz)), aus Fachbeamten (Manfred Kopf, Andreas Marlin und Stefan Obkircher aus der Abteilung Raumplanung der Vorarlberger Landesregierung) und aus Vertretern der Zivilgesellschaft (Peter Haßlacher und Josef Essl von der Internationalen Alpenschutzkommission CIPRA-Österreich (Innsbruck)).

Alle Mitglieder der Arbeitsgruppe zeichnen sich dadurch aus, dass sie sich seit vielen Jahren mit der Alpenentwicklung und den Problemen des Freiraumschutzes beschäftigen und sich dabei für unterschiedliche Lösungsansätze engagieren. Damit handelt es sich um eine Arbeitsgruppe, deren Zusammensetzung und Aufgabenstellung alle Anforderungen an ein ,transdisziplinäres“ Vorgehen im Sinne von Mittelstraß (1998: 45 f.) erfüllt.

Die Arbeit der Arbeitsgruppe und die vorliegende Veröffentlichung sind durch vier normative Leitideen geprägt: 
1. Es geht nicht um Freiräume im Dauersiedlungsbereich, sondern um solche im Außenbereich, also um Gebiete oberhalb der Dauersiedlungen, die etwa an der Untergrenze des geschlossenen Waldes beginnen. Damit ist die normative Zielsetzung verbunden, dass eine bessere Vernetzung der Lebensräume zwischen Bergwäldern, Lichtalmen und hochalpinem Ödland erreicht werden soll (S. 3).

2. Es wird nur der deutschsprachige Alpenraum thematisiert, genauer: die Alpen in Bayern, in den österreichischen Bundesländern Salzburg, Tirol, Vorarlberg, in der Schweiz und in Südtirol. Diese räumliche Auswahl wird getroffen, weil in diesen Alpenräumen der Bevölkerungsund Nutzungsdruck besonders groß sind und weil es hier wichtige kulturelle und politische Gemeinsamkeiten gibt, auf die man sich bei Lösungen beziehen möchte (S. 3).

3. Als Freiraum werden keine Flächen verstanden, die gänzlich frei von Nutzung sind, sondern die durchaus gewisse Nutzungsstrukturen besitzen können (sakrale Bauten, Gipfelkreuze, Brunnen, Denkmäler, schmale Wege u. Ä.), die aber keinen ,störenden“ Charakter annehmen dürfen und die ,lärmfrei“ sind (S. 9). Diese normative Definition leitet sich aus einem Verständnis der Alpen als Natur- und Kulturlandschaft ab (S. 1), grenzt sich explizit gegen die aktuelle „Wildnis“-Diskussion ab (S. 9 und 66) und legt das Schwergewicht auf „,die Zugänglichkeit und Erlebbarkeit dieser Räume“ (S. 9), um so „die Akzeptanz für Freiräume“" zu steigern (S. 9 und 66).

4. Als Lösungsrichtung werden ausschließlich Instrumente der Raumordnung thematisiert und diskutiert, was mit der Forderung nach einem größeren Stellenwert der Raumordnung und der Ausarbeitung von „,neuen Wegen“ in der Raumordnung verbunden wird (S. 3 f.).

Diese vier normativen Leitideen grenzen die überkomplexe Leitfrage auf eine sinnvolle Weise ein und bilden so die Grundlage für eine produktive Arbeit. Die konkreten Arbeitsschritte, die daraus folgen, umfassen zuerst die Darstellung des Forschungsstandes und die Erarbeitung einer Definition von Freiraum (Kapitel 2). Dann folgen Forschungsdesign und Methodik (Kapitel 3) sowie die Analyse der supranationalen Vorgaben (Alpenkonvention und EU Strategy for the Alpine Region - EUSALP, Kapitel 4) und die der bestehenden Instrumente zum Erhalt von Freiräumen (Kapitel 5 und 6). Die Diskussion der Ergebnisse bildet den Abschluss (Kapitel 7). Die detaillierte Darstellung der Kapitel 4 bis 6 stellt den zentralen Kern der Publikation dar.

\section{Zur Einordnung und Bedeutung dieses Forschungsprojektes}

Wie die Autoren selbst schreiben (S. 3), lag und liegt der Fokus bei den Diskussionen über Freiräume in der Regel auf dem Dauersiedlungsraum. Klassisches Beispiel dafür sind der immer noch bekannte Buchtitel von Jost Krippendorf „Die Landschaftsfresser“ (1975) oder die aktuellen Diskussionen in der Schweiz um Zweitwohnungen. Und wenn heute Freiräume oberhalb der Dauersiedlungen thematisiert werden, dann ist dies in der Regel mit der Perspektive der „Wildnis“-Gebiete verbunden. Diese Publikation setzt dagegen ganz bewusst auf einen anderen Ansatz und öffnet damit auf innovative Weise neue Wege für Lösungsmöglichkeiten durch Instrumente der Raumordnung. Damit steht dieser Ansatz quer zu den heute üblichen Ansätzen, und deshalb wirkt er so erfrischend und so innovativ. Es ist daher unbedingt zu wünschen, dass er innerhalb und außerhalb der Alpen breit wahrgenommen und intensiv diskutiert wird, denn er hat die Qualität, die laufenden Diskussionen auf eine produktive Weise zu bereichern.

\section{Zur Bewertung dieses Ansatzes}

Der gesamte Argumentationsgang dieser Publikation ist nachvollziehbar dargestellt und inhaltlich stringent entwickelt. Der materialreiche Hauptteil mit der Analyse der unterschiedlichen Instrumente für Freiraumsicherung fällt sehr informativ aus und stellt in seiner Übersichtlichkeit ein wichtiges Hilfsmittel für die aktuellen Diskussionen dar. Und der Schluss skizziert ein anwendungsorientiertes und stimmiges raumordnerisches Vorgehen zur langfristigen Sicherung der Freiflächen in den Alpen. Lediglich an den folgenden Punkten gibt es Diskussionsbedarf:

Die Einleitung thematisiert zwar die zentralen normativen Voraussetzungen, aber dabei wird das Verständnis von Freiräumen (keine Wildnis-Gebiete) nicht angesprochen, obwohl dies an dieser Stelle wichtig gewesen wäre. Dies wird zwar in Kapitel 2 „Freiräume“ nachgeholt, aber eigentlich wird erst zu Beginn von Kapitel 7 auf S. 66 so richtig deutlich, welchen Stellenwert dieses Freiraumverständnis hier besitzt. Und die sehr detaillierten Festlegungen am Ende von Kapitel 2.2 (S. 9) wirken an dieser Stelle etwas konkretistisch und wären besser in Kapitel 7 aufgehoben.

Die zentrale Forderung, eine langfristige Sicherung alpiner Freiräume wäre nur mit Instrumenten der Raumordnung erreichbar, wird zwar immer wieder betont, aber eine vergleichende Auseinandersetzung mit möglichen alternativen Instrumenten wie Naturschutz- und anderen sektoralen Politikinstrumenten oder rechtlichen und politischen Festlegungen unterschiedlichster Art wird nicht durchgeführt. 
Dadurch hängt der zentrale Teil der Argumentation etwas in der Luft.

Es macht den Eindruck, als wäre diese Arbeitsgruppe als Reaktion auf den Konflikt um die Skierschließung des Riedberger Horns im Allgäu entstanden, in dessen Verlauf die Bayerische Staatsregierung kürzlich die Festlegungen des Alpenplans zum ersten Mal seit seinem Bestehen aufweicht. Es liegt nahe, gerade in dieser Situation eine stärkere Raumordnung zu fordern. Natürlich ist der Gedanke sehr bestechend, in einer Zeit, in der ,der neoliberale Zeitgeist in der heutigen Politik" (S. 4) alle noch bestehenden Schranken für eine unbegrenzte Wirtschaftsdynamik niederreißt, mit der Raumordnung die Aufgabe ,Ordnung' gegen die Aufgabe ,Entwicklung' stark zu machen und so wieder ein Gleichgewicht der Kräfte herstellen zu wollen. Aber wer soll die so wichtige Funktion der Ordnung politisch durchsetzen? Dies erscheint heute schwerer denn je, da die Kräfte an der Basis derzeit schwach sind, aber auch zentralistische Lösungen nicht unbedingt eine Alternative darstellen. Der
Rezensent bekennt, dass er darauf ebenfalls keine Antwort hat. Aber er fragt sich, ob es in einer solchen Situation sinnvoll ist, mit dem Instrument der Raumordnung bereits eine prinzipielle Antwortrichtung vorzudenken, oder ob es nicht vielleicht sinnvoller wäre, sich ganz auf das Ausgangsproblem zu konzentrieren, anhand exemplarischer Problemfälle möglichst viele Kräfte zu bündeln, dabei konkrete, pragmatische Antworten zu finden und dann zu sehen, ob sich daraus eventuell neue prinzipielle Lösungen entwickeln?

Es ist ein großes Verdienst dieser Publikation, dass sie diese Grundsatzfragen provoziert, und auch deshalb ist ihr eine weite Verbreitung zu wünschen.

\section{Literatur}

Krippendorf, J. (1975): Die Landschaftsfresser: Tourismus und Erholungslandschaft, Verderben oder Segen? Bern.

Mittelstraß, J. (1998): Die Häuser des Wissens. Wissenschaftstheoretische Studien. Frankfurt am Main. 